\section{IMMUNOTHERAPY RELATED ADVERSE EVENTS: A SINGLE CENTER EXPERIENCE}

Wei Yang*, Julie Rowe, Sophia Lee, Jing Zhang, Mohammad Rahbar. University of Texas @ Houston, Houston, TX, United States

Background As the role of immune checkpoint inhibitors (ICIs) expands in many malignancies, including hepatocellular carcinoma (HCC), a better understanding of the predictors of immunotherapy-related adverse events (irAEs) is needed due to the complexity that ICIs add to patient care. ${ }^{1-5}$

Methods We conducted a single-institution retrospective chart review for cancer patients of any type who received at least one ICI between January 2015 and December 2020. Demographic, social, cancer-related, laboratory, and treatment variables were collected, along with irAE data. Exploratory statistical analysis was performed to find predictors of increased irAEs.

Results A total of 342 patients were included in the study: 133 women and 209 men. Median age was 65 years. The most common cancers were lung $(110,32.07 \%)$, kidney (51, $14.87 \%)$, and HCC $(43,12.54 \%)$. All patients received at least one dose of ICI (table 1); 11 received combination ICIs. One hundred and two (26.53\%) patients developed irAEs of any grade (table 2 ). Nineteen patients $(5.56 \%)$ had a grade 3 or 4 irAE; 20 patients required systemic steroids. No biologics were used for the management of severe irAEs. Patients who received prior chemotherapy were less likely to develop irAEs (odds ratio $[\mathrm{OR}]=0.42, \mathrm{p}=0.0006$ ). A history of hyperthyroidism or hypothyroidism was associated with more irAEs $(\mathrm{p}=0.011)$. Combination ICI led to more irAEs overall (OR $=2.91, \mathrm{p}=0.043)$, as well as grade 3 or 4 events $(\mathrm{OR}=$ $5.32, \mathrm{p}=0.008)$. A trend toward more grade 3 or 4 events occurred in HCC patient $(\mathrm{OR}=2.78, \mathrm{p}=0.06)$. Older patients showed a trend toward more irAEs $(p=0.08)$. Worse peri-treatment renal function was associated with an increased risk of irAE $(\mathrm{OR}=1.86, \mathrm{p}=0.047)$. A higher peri-treatment hemoglobin nadir was associated with a lower risk of irAE $(\mathrm{OR}=0.45, \mathrm{p}=0.07)$. Several other variables had ORs or confidence intervals close to 1 , including number of sessions of ICI and higher baseline AST.

Conclusions Prior chemotherapy, worse renal function, and thyroid dysfunction were associated with irAEs, whereas higher hemoglobin nadir was protective against irAE. Unlike the current literature, our study included a large number of HCC patients. The higher irAE incidence in our study could be associated with this higher number of HCC patients; however, further studies are needed.

Abstract 807 Table 1 Immune checkpoint inhibitors received

\begin{tabular}{|l|r|r|}
\hline Therapy & No. & \multicolumn{1}{c|}{ Percent } \\
\hline Nivolumab & 200 & $58.31 \%$ \\
\hline Pembrolizumab & 137 & $39.94 \%$ \\
\hline Ipilimumab & 21 & $6.12 \%$ \\
\hline Durvalumab & 9 & $2.62 \%$ \\
\hline Atezo & 8 & $2.33 \%$ \\
\hline Ipilimumab + Nivolumab & 11 & $3.22 \%$ \\
\hline
\end{tabular}

Abstract 807 Table 2 irAE incidence

\begin{tabular}{|l|r|r|}
\hline irAE & \multicolumn{1}{l}{ Count } & \multicolumn{1}{l|}{ Percent } \\
\hline Endocrine & 43 & $12.54 \%$ \\
\hline Gastrointestinal & 33 & $9.62 \%$ \\
\hline Skin & 17 & $4.96 \%$ \\
\hline Rheumatologyc & 5 & $1.46 \%$ \\
\hline Pulmonary & 3 & $0.87 \%$ \\
\hline Musculoskeletal & 1 & $0.29 \%$ \\
\hline Renal & 1 & $0.29 \%$ \\
\hline Eye & 0 & $0.00 \%$ \\
\hline CNS & 0 & $0.00 \%$ \\
\hline Cardiovascular & 0 & $0.00 \%$ \\
\hline Total with irAE & 102 & $29.74 \%$ \\
\hline
\end{tabular}

\section{REFERENCES}

1. Brahmer JR, et al. Management of immune-related adverse events in patients treated with immune checkpoint inhibitor therapy: American Society of Clinical Oncology Clinical Practice Guideline. JCO 2018;36:1714-1768.

2. National Comprehensive Cancer Network. Management of Immunotherapy-Related Toxicities, 2021. https://www.nccn.org/professionals/physician_gls/pdf/immunotherapy.pdf

3. Kartolo A, Sattar J, Sahai V, Baetz T, Lakoff JM. Predictors of immunotherapyinduced immune-related adverse events. Curr Oncol 2018;25:e403-e410.

4. Suresh $K$, et al. Pneumonitis in non-small cell lung cancer patients receiving immune checkpoint immunotherapy: incidence and risk factors. Journal of Thoracic Oncology 2018;13:1930-1939.

5. Colen RR, et al. Radiomics to predict immunotherapy-induced pneumonitis: proof of concept. Invest New Drugs 2018;36:601-607.

Ethics Approval The study involving retrospective review of patient records was approved under the Institutional Review Board. All records identifying the patient was be kept confidential and, to the extent permitted by the applicable laws and/or regulations, were not be made publicly available. Patient names will not be supplied to third parties. A unique study number will be assigned to each patient. Study data stored electronically will be stored in a password-protected, encrypted computers. Paper study data will be maintained by the primary investigators in the locked research offices.

Consent N/A

http://dx.doi.org/10.1136/jitc-2021-SITC2021.807 